\title{
ON THE VOLUME OF MANIFOLDS ALL OF WHOSE GEODESICS ARE CLOSED
}

\author{
ALAN WEINSTEIN
}

\section{1. $C_{L}$-manifolds}

A riemannian manifold $(M, g)$ will be called a $C_{L}$-manifold if all the geodesics on $M$ are closed and have length $2 \pi L$, i.e., if all the orbits of the geodesic flow on the unit tangent bundle $U(M, g)$ are periodic with least period $2 \pi L$. It is a problem of some interest to characterize these manifolds, which are the "simple harmonic oscillators" of riemannian geometry.

The best known examples of $C_{L}$-manifolds are the symmetric spaces of rank one, or $S C$-manifolds, as they are called by Berger [1, III. 4]. These are the spheres $\left(S^{n}\right.$, can $)$, projective spaces $\left(P^{n}(K)\right.$, can) for $k=\boldsymbol{R}, \boldsymbol{C}$, or $\boldsymbol{H}$, and the Cayley projective plane $\left(P^{2}(\Gamma)\right.$, can), with their canonical metrics. The spheres are $C_{1}$-manifolds, and the projective spaces are, with Berger's normalization, $C_{1 / 2}$-manifolds.

Zoll (see [1, IV. 8]) in 1903 constructed examples of non-standard $C_{L}$-metrics on $S^{2}$ (surfaces of revolution), and Blaschke [3, p. 233] gives an example, due to Thomsen, of a $C_{L}$-metric on $S^{2}$ with no nontrivial isometries. These constructions can be carried out on higher dimensional spheres as well. If one strengthens the $C_{L}$ condition to require that the geodesics be simple closed curves on $M$, then a theorem of Green (see [1, VIII. 9]) states that any such simple $C_{L}$-metric on $P^{2}(R)$ has constant curvature. Furthermore, it is a theorem of Bott (see [1, IV.6]) that every simple $C_{L^{-}}$-manifold has the same integer cohomology ring as some $S C$-manifold. In fact, this result requires only that all the geodesics through a single point of $M$ be simply closed with the same length. The earliest topological study of $C_{L}$-manifolds seems to be that of Reeb [7], who proved, among other things, that the product of two spheres of different odd dimensions cannot carry a $C_{L}$-metric.

The aim of the present paper is to demonstrate the following geometric result.

Theorem A. If $(M, g)$ is an $n$-dimensional $C_{L}$-manifold, then the ratio

$$
i(M, g)=\frac{\operatorname{vol}(M, g)}{L^{n} \operatorname{vol}\left(S^{n}, \mathrm{can}\right)}
$$

is an integer.

Communicated by F. Hirzebruch, May 18, 1973. 
We will actually prove the following theorem, of which Theorem A is an immediate consequence.

Theorem B. If $(M, g)$ is an n-dimensional $C_{L}$-manifold, then the real number $j(M, g)$ defined by the equation

$$
\operatorname{vol}(M, g)=\frac{(2 \pi L)^{n} \cdot j(M, g)}{(n-1) ! \operatorname{vol}\left(S^{n-1}, \mathrm{can}\right)}
$$

is an even integer.

To prove Theorem A from Theorem B, one has merely to check, using the values of $\operatorname{vol}\left(S^{n-1}\right.$, can $)[1$, VI. 7$]$, that $j\left(S^{n}\right.$, can $)=2$; then set $i(M, g)=$ $\frac{1}{2} j(M, g)$.

\section{Remarks}

1. The proof of Theorem B, contained in the following two sections of this paper, identifies the integer $j(M, g)$ as a topological invariant of the fibration of $U(M, g)$ by the orbits of the geodesic flow.

2. Using Gysin sequences one can prove that $j(M, g)=2$ and $i(M, g)=1$ if $M$ is an even-dimensional sphere. It would be interesting to prove that $i(M, g)$ is independent of $g$ when $M$ is any $S C$-manifold. This may be a step in the direction of generalizing the theorem of Green mentioned above.

3. In the succeeding paper in this journal [2], Marcel Berger proves the following application of Theorem A. Let $g$ be a Kählerian metric on $P^{n}(C)$, compatible with the standard complex structure. Suppose that the distance to the first conjugate point in each direction from each point on $P^{n}(C)$ is $\frac{1}{2} \pi$. Then, at least if $g$ is sufficiently near the canonical metric in the $C^{0}$ topology, $\left(P^{n}(C), g\right)$ is isometric to $\left(P^{n}(C)\right.$, can $)$.

4. Funk $\left[4\right.$, p. 283] remarks that the area of a $C_{1}$-surface of revolution must be $4 \pi$. Otherwise, our result seems to be new even for $M=S^{2}$.

5. An amusing consequence of Theorem $\mathrm{A}$ is that one cannot apply a slight perturbation to $\left(S^{n}, \mathrm{can}\right)$ to make the geodesics close only after $k>1$ "revolutions", for then the volume of the manifold would have to be multiplied by $k^{n}$.

6. For reference, we present the following formulas, obtained from the calculations in [1, VI. 7]:

$$
\begin{gathered}
i\left(S^{n}, \text { can }\right)=1, \quad i\left(P^{n} \boldsymbol{R}, \text { can }\right)=2^{n-1}, \\
i\left(P^{n} C, \text { can }\right)=\left(\begin{array}{r}
2 n-1 \\
n-1
\end{array}\right), \quad i\left(P^{n} H, \text { can }\right)=\frac{1}{2 n+1}\left(\begin{array}{c}
4 n-1 \\
2 n-1
\end{array}\right), \\
i\left(P^{2} \Gamma, \text { can }\right)=39 .
\end{gathered}
$$

7. The work described here was carried out at the Institute for Advanced 
Study, Princeton and Institut des Hautes Études Scientifiques, Bures-sur-yvette with support from a Sloan fellowship and was completed in Berkeley, with support from NSF grant GP-34785X. The author would like to thank M. Berger and F. Hirzebruch for their helpful comments.

\section{Proof of Theorem $B$}

The unit tangent bundle $U(M, g)$ of a riemannian manifold carries the following geometric objects:

the geodesic spray $G, \quad[1$, IV. 2],

the canonical one-form $\alpha$, [1, III. 6],

the canonical two-form $d \alpha$, [1, III. 6],

the riemannian metric $\overline{\bar{g}}, \quad[1, \mathrm{~V} .2 .4]$,

the volume element $\overline{\bar{\theta}}, \quad[1, \mathrm{~V} .2 .4]$.

These objects satisfy the following relations:
i. $\left|\frac{\alpha \wedge(d \alpha)^{n-1}}{(n-1) !}\right|=\overline{\bar{\theta}}, \quad[1, \mathrm{~V} .2 .5]$,
ii. $\operatorname{vol}(U(M, g), \overline{\bar{g}})=\operatorname{vol}(M, g) \cdot \operatorname{vol}\left(S^{n-1}, \operatorname{can}\right), \quad[1, \mathrm{~V} .2 .13]$,
iii. the flow of $G$ leaves $\alpha$ invariant [1, IV. 3.10],
iv. $\alpha(G) \equiv 1, \quad[1$, p. 125],
v. the null space (characteristic distribution) of $d \alpha$ is generated by $G$, [5, Thm. 5.9].

Since the orbits of $G$ are all periodic with period $2 \pi L$, the vector field $2 \pi L G$ generates a free action of $S^{1}=\boldsymbol{R} / \boldsymbol{Z}$ on $U(M, g)$, with quotient a manifold $C(M, g)$. The projection $U(M, g) \stackrel{p}{\longrightarrow} C(M, g)$ is a principal bundle with structure group $S^{1}$. Relations iii and iv above mean that $\alpha /(2 \pi L)$ is a connection form on this bundle, and $d \alpha /(2 \pi L)$ is the curvature form. There is then a uniquely determined form $\Omega$ on $C(M, g)$ such that $p^{*} \Omega=d \alpha /(2 \pi L)$; the de Rham cohomology class $[\Omega] \in H^{2}(C(M, g) ; R)$ is the image under the coefficient homomorphism $\rho_{2}: H^{2}(C(M, g) ; Z) \rightarrow H^{2}(C(M, g) ; R)$ of the Euler class $e(p)$ of the bundle $p$. (We identify the group $S^{1}$ with $S O(2)$.) Then $\left[\Omega^{n-1}\right]=[\Omega]^{n-1}$ is the image of $[e(p)]^{n-1}$ under the coefficient homomorphism $\rho_{2 n-2}$.

By relation $\mathrm{v}$, the form $\Omega$ is nonsingular on $C(M, g)$, which is oriented by $\Omega^{n-1}$. Denoting by $[C(M, g)]$ the fundamental $(2 n-2)$-cycle, we have

$$
\int_{C(M, g)} \Omega^{n-1}=\left\langle[e(p)]^{n-1},[C(M, g)]\right\rangle .
$$

Let $j(M, g)$ be the quantity on either side of (2). The left hand side of the equation is positive, and the right hand side is an integer, so $j(M, g)$ is a positive integer.

The argument up to here is essentially contained in [7]. At this point, we use the Fubini theorem for fibrations $[1,0.3 .17]$ to calculate 


$$
\begin{aligned}
\operatorname{vol}(U(M, g) ; \overline{\bar{g}}) & =\int_{U(M, g)} \frac{\alpha \wedge(d \alpha)^{n-1}}{(n-1) !} \\
& =\frac{1}{(n-1) !} \int_{U(M, g)} \alpha \wedge p^{*}(2 \pi L \Omega)^{n-1} \\
& =\frac{(2 \pi L)^{n-1}}{(n-1) !} \int_{x \in C(M, g)}\left[\int_{p^{-1}(x)} \alpha\right] \Omega^{n-1}
\end{aligned}
$$

By relation iv, $\int_{p^{-1}(x)} \alpha=2 \pi L$ for each $x$, so the above expression becomes

$$
\frac{(2 \pi L)^{n}}{(n-1) !} \int_{C(M, g)} \Omega^{n-1}=\frac{2 \pi L}{(n-1) !} j(M, g) .
$$

Combining this with relation ii shows that $j(M, g)$ satisfies (1). To complete the proof of Theorem B, it remains only to show that $j(M, g)$ is even. This is done in the next section.

\section{Involutions and evenness}

Let $\xi: P \rightarrow B$ be a principal bundle with structure group $S O(2)$. By means of the embedding $S O(2) \rightarrow O(2)$, we can consider $\xi$ as a bundle with fibre $S O(2)$ and structure group $O(2)$. Let $\beta: \xi \rightarrow \xi$ be a mapping of $O(2)$ bundles (see $[8,2.5]$ for a definition) with $\beta^{2}=$ identity and such that the induced mapping $\gamma: B \rightarrow B$ has no fixed points. Suppose further that $B$ is an orientable manifold of dimension $2 n$.

Proposition. The class $[e(\xi)]^{n}$ is an even multiple of the generator of $H^{2 n}(B ; Z) \approx Z$.

Proof. By $[6,4.11 .2 \mathrm{III}],[e(\xi)]^{n}=e(n \xi)$ where $n \xi$ is the $S O(2 n)$ bundle obtained by taking the $n$-fold Whitney sum of $\xi$ with itself. The involution $\beta$ induces an involution $n \beta: n \xi \rightarrow n \xi$ of $O(2 n)$ bundles; therefore there is an $O(2 n)$ bundle $\overline{n \xi}$ over the quotient manifold $\bar{B}=B / \gamma$ such that $n \xi=\pi^{*} \overline{n \xi}$, $\pi: B \rightarrow \bar{B}$ being the projection. The Whitney classes of $n \xi$ and $\overline{n \xi}$ satisfy the relation $w_{2 n}(n \xi)=\pi^{*} w_{2 n} \overline{(n \xi)}[6$, p. 73]. Since $\pi$ is a double covering, it induces the zero map from $H^{2 n}\left(\bar{B} ; Z_{2}\right)$ to $H^{2 n}\left(B ; Z_{2}\right)$, so $w_{2 n}(n \xi)=0$. But $w_{2 n}(n \xi)$ is the $\bmod 2$ reduction $[6, \text { p. 73] of } e(n \xi)=e(\xi)]^{n}$, so $[e(\xi)]^{n}$ is even. q.e.d.

To apply this Proposition to Theorem 2, we use the involution $h_{-1}: U(M, g)$ $\rightarrow U(M, g)$ defined by multiplying each tangent vector by -1 . Since $G$ is a spray, $G$ is $h_{-1}$-related to $-G$, so $h_{-1}$ is an $O(2)$ bundle mapping. Finally, the induced map on $C(M, g)$ has no fixed points, because a geodesic cannot double back upon itself in the reverse direction.

Hence the class $[e(p)]^{n-1}$ is an even multiple of the generator of $H^{2 n-2}(C(M$, $g) ; Z)$, and $j(M, g)=\left\langle[e(p)]^{n-1},[C(M, g)]\right\rangle$ is an even integer. 


\section{References}

[1] M. Berger, Lectures on geodesics in riemannian geometry, Tata Institute of Fundamental Research, Bombay, 1965.

[2] - Sur certaines variétés kähleriennes a géodésiques toutes fermées, J. Differential Geometry 9 (1974) 519-520.

[ 3 ] W. Blaschke, Vorlesungen über Differentialgeometrie, 3rd. edition, Springer, Berlin, 1930.

[4] P. Funk, Über Flächen mit lauter geschlossenen geodätischen Linien, Math. Ann. 74 (1913) 278-300.

[5] C. Godbillon, Géométrie différentielle et mécanique analytique, Hermann, Paris, 1969.

[ 6] F. Hirzebruch, Topological methods in algebraic geometry, Third enlarged edition (translated by R. L. E. Schwarzenberger), Springer, New York, 1966.

[ 7 ] G. Reeb, Variétés de Riemann dont toutes les géodésiques sont fermées, Acad. Roy. Belg. Bull. Cl. Sci. (5) 36 (1950) 324-329.

[ 8 ] N. Steenrod, The topology of fibre bundles, Princeton University Press, Princeton, 1951.

UNIVERSITY OF CALIFORNIA, BERKELEY 
